\title{
Molecular genetics of medulloblastoma in children: diagnostic, therapeutic and prognostic implications
}

\author{
Maher El Doussouki ${ }^{1}$, Amar Gajjar ${ }^{2,3}$ \& Omar Chamdine*,1 \\ ${ }^{1}$ Department of Pediatric Hematology Oncology, King Fahad Specialist Hospital, Dammam, Kingdom of Saudi Arabia \\ ${ }^{2}$ Department of Oncology, St Jude Children's Research Hospital, Memphis, TN, USA \\ ${ }^{3}$ Department of Pediatrics, University of Tennessee Health Science Center, Memphis, TN, USA \\ *Author for correspondence: Tel.: +96 654056 6897; omar.chamdine@kfsh.med.sa
}

Medulloblastoma is the most common embryonal tumor in children. The current standard of care comprises surgical resection, radiation and chemotherapy. Patients are stratified into standard and high risk based on the degree of resection, presence of metastatic disease and histopathology. Cure rates dramatically improved during the past decades reaching $70-80 \%$ (high and average risk, respectively). Infant medulloblastoma has a worse outcome as the use of radiation therapy is very limited, a group of patients still has dismal outcome despite appropriate therapy, and the unacceptable long-term therapy side effects in survivors. Advanced molecular techniques have allowed scientists to discover four distinct molecular subgroups and correlate them with multiple factors such as histopathology, clinical behavior and possible therapeutic targets.

First draft submitted: 3 September 2018; Accepted for publication: 4 December 2018; Published online: 25 January 2019

Keywords: classification $\bullet$ epigenetics $\bullet$ medulloblastoma $\bullet$ molecular genetics $\bullet$ recurrence $\bullet$ SHH $\bullet$ SMO inhibitor - subgroup • targeted therapy $\bullet$ WNT

Medulloblastoma (MB) is the most common malignant brain tumor (BT) in children. It comprises up to $20 \%$ of all pediatric BT and $64 \%$ of all embryonal BT [1]. It is commonly diagnosed before 19 years of age and has a male predominance [1,2]. Progress in MB classification, diagnosis, treatment and prognosis reflects a fascinating journey in which survival has improved from near zero to as high as $80 \%$ (Figure 1). This has been achieved with better understanding of the disease histology and incorporation of multimodality treatment including surgery, radiation and chemotherapy [3,4]. Patients are currently classified into standard and high risk based on postoperative residual disease ( $>1.5$ and $<1.5 \mathrm{~cm}^{2}$ ), histology and metastatic disease at diagnosis [5,6]. Young age is also prognostic as children $<3-5$ years of age do not receive craniospinal irradiation further compromising their outcome $[7,8]$. This success was not without a cost. Long-term survivors, especially young children ( $<5$ years of age), developed multiple complications, most important of which are the devastating effects on the IQ levels and cognitive function. Other complications included endocrinological, hearing, fertility, cardiopulmonary fitness and physical performance problems, in addition to the risk of secondary malignancies [9-12].

Since the early 1990 's, cumulative findings have gradually led to the discovery of molecular characteristics in medulloblastoma and supported the classification into four molecular subgroups (Wingless [WNT], Sonic Hedgehog [SHH], group 3 [G3] and group 4 [G4]). This was reflected in the consensus conference in Boston in 2010 and became internationally accepted following the publication of Taylor and colleagues in 2012 [6,13-21]. In the following years, a massive amount of genomic characterization using advanced molecular techniques has been conducted on hundreds of primary tumors by several independent groups, and each year multiple publications emerge, either introducing new findings in the field or refining earlier ones by discovering substructures within each group reflecting distinct clinicopathological and molecular features $[11,20-33]$. This has been largely possible due to parallel advances in genetic testing technologies, such as expression profiling, whole-genome sequencing, whole

Future Medicine 


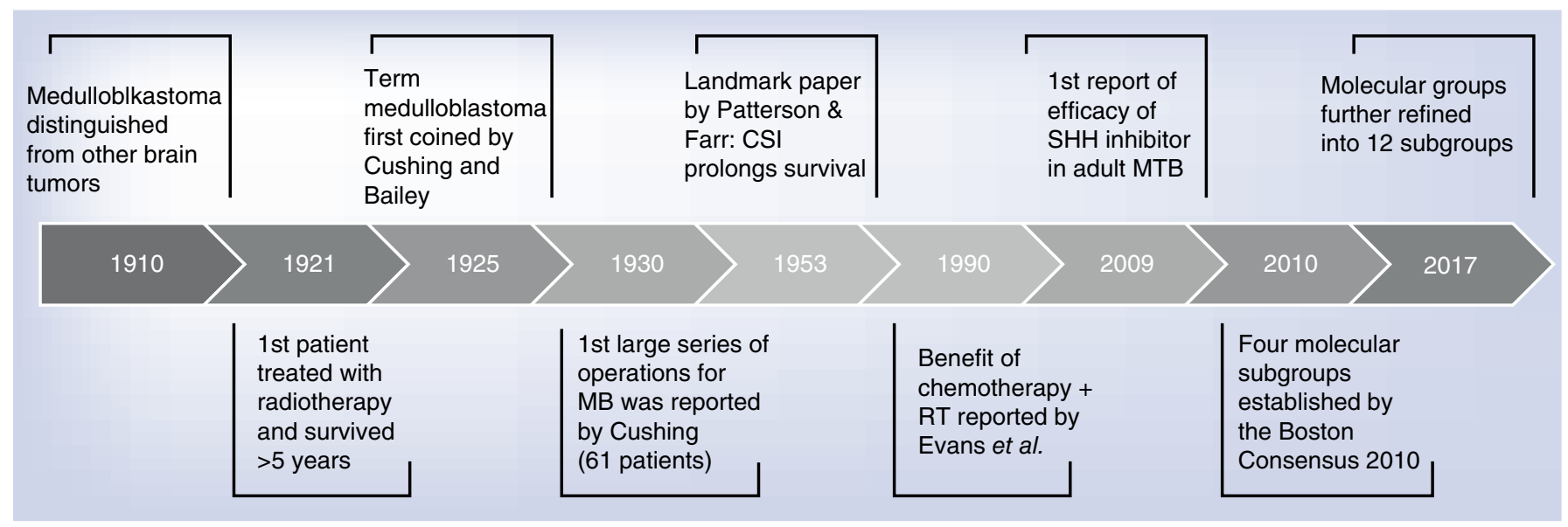

Figure 1. Historic timeline of medulloblastoma progress.

exome sequencing and methylation profiling, among other technologies [16,34]. In 2016, WHO Classification of Tumors of the Central Nervous System incorporated the MB molecular subgroups into the MB classification [35].

In addition to genetic alterations, epigenetic deregulation occurs in $30-40 \%$ of $\mathrm{MB}$, declaring itself as major driver in this disease $[33,36,37]$. This review will focus on the molecular characteristics of MB in children and their implications on various aspects of the disease (see Table 1 for summary).

\section{WNT-subgroup}

The WNT is a family of growth factor receptors that are involved in embryogenesis and in cell-cell control mechanisms $[17,38]$. WNT $/ \beta$-catenin signaling is a highly conserved pathway that regulates key cellular functions including proliferation, differentiation, migration, genetic stability, apoptosis and stem cell renewal. Aberrations in this pathway are implicated in several cancers including colon cancer, breast cancer, adrenocortical tumor, melanoma, high grade glioma and MB [38].

The WNT subgroup is the least common among the $4 \mathrm{MB}$ molecular subgroups, comprising around $10-15 \%$ of the cases. It can occur at any age; however, it is most commonly encountered in children 6-12 years of age (peak incidence of 10-12 years) with slight female predominance $[13,21,30,34,39,40]$. Occurrence in children less than 4 years old is extremely uncommon $[21,36,41]$. The vast majority of WNT tumors are of classic histology; only rare cases are of large cell/anaplastic (LCA) variant and are never nodular desmoplastic (ND). Metastatic disease is encountered in less than $10 \%$ of newly diagnosed cases $(5-10 \%)[36,40,42]$. The lower rhombic lip progenitor cells of the dorsal midbrain have been proven to be the cells of origin in WNT tumors. This is supported by the fact that anatomically, WNT MB often develop in a central location, frequently abutting the brainstem and protruding through the foramen of Lushcka $[28,34,43]$. Total of $85-90 \%$ of WNT MB cases harbor mutations in CTNNB1, the gene encoding for the protein $\beta$-catenin. Mutant $\beta$-catenin protein is resistant to degradation, leading to its accumulation in the cell nucleus [42]. Whole-genome sequencing has identified recurrent somatic mutations in WNT MB other than CTNNB1 gene. The most prevalent among those genes are DDX3X, SMARCA4, TP53 and KMT2D. These gene mutations are found in approximately 50,26, 15 and $12 \%$ respectively, and they are not exclusive to WNT MB. The DDX3X mutations are found in $11 \%$ of $\mathrm{SHH} \mathrm{MB}$ and $3 \%$ of group $3 \mathrm{MB}$, SMARCA4 is a crucial component of the SWI/SNF pathway, which has a major role in the development of multiple cancers including ovarian cancer, melanoma, renal and liver cancer as well as other tumors. The TP53 mutations are found in $14 \%$ of $\mathrm{SHH} \mathrm{MB}$, and KMT2D is encountered in $13 \%$ of $\mathrm{SHH} \mathrm{MB}$ and $4 \%$ of group $3 \mathrm{MB}$. These genes encode proteins that interact with nuclear $\beta$-catenin and remodel chromatin, suggesting that cooperative mutations occur in the development of this tumor subtype $[13,21,28,30,40,42,44]$. Monosomy 6 is found in $80-85 \%$ of WNT MB, which is strongly associated with a WNT pathway immunohistochemical profile [45,46]. It is a hallmark chromosomal aberration of WNT MB and is rarely seen in other subgroups although a minority of tumors retain two copies of the chromosome $[36,42,45,47]$. The WNT MB subgroup is most accurately diagnosed by sequencing exon 3 of CTNNB, DNA methylation profiling or gene expression profiling [48,49]. These methods are expensive 
Table 1. Summary of medulloblastoma demographics and molecular characteristics by molecular subgroup.

\begin{tabular}{|c|c|c|c|c|}
\hline Subgroup & WNT & SHH & G3 & G4 \\
\hline Frequency & $10-15 \%$ & $28-30 \%$ & $25-28 \%$ & $40-45 \%$ \\
\hline Age range (years) & $\begin{array}{l}6-12 \text { (peaks at } 10-12) \\
>17 \text { ( } \beta \text { subgroup) }\end{array}$ & Bimodal $(<4,>16)$ & Infants and young children & All age groups (median 9) \\
\hline Histology & $\begin{array}{l}\text { Mostly classic, rarely } \\
\text { LCA, never ND }\end{array}$ & Mostly ND, classic \& LCA (less common) & Classic (most common), LCA & Classic and LCA (less common) \\
\hline Cell of origin & $\begin{array}{l}\text { Lower rhombic lip } \\
\text { progenitor cells of the } \\
\text { dorsal midbrain }\end{array}$ & Cerebellar GCP of the EGC & $\begin{array}{l}\text { Cerebellar GCP of the EGC (prominin } \\
1+/ \text { lineage-neural stem cells) }\end{array}$ & Unknown \\
\hline Subgroups & $\alpha \& \beta$ & $\alpha, \beta, \gamma \& \delta$ & $\alpha, \beta \& \gamma$ & $\alpha, \beta \& \gamma$ \\
\hline $\begin{array}{l}\text { Anatomic } \\
\text { location }\end{array}$ & $\begin{array}{l}\text { Central, frequently } \\
\text { abutting brainstem and } \\
\text { infiltrating foramen of } \\
\text { Lushcka }\end{array}$ & Hemispheric (rarely midline) & $\begin{array}{l}\text { Midline (filling fourth ventricle), } \\
\text { hemispheric cases reported }\end{array}$ & Midline (filling fourth ventricle) \\
\hline $\begin{array}{l}\text { Metastatic } \\
\text { disease at } \\
\text { diagnosis }\end{array}$ & $8.6 \%(\alpha) \quad 21.4 \%(\beta)$ & $20 \%(\alpha) \quad 33 \%(\beta)$ & $43.4 \%(\alpha) \quad 20 \%(\beta) \quad 39.4 \%(\gamma)$ & $40 \%(\alpha) \quad 40.7 \%(\beta) \quad 38.7 \%(\gamma)$ \\
\hline IHC & Nuclear $\beta$-catenin & Filamin A, YAP1, SFRP1, GAB1 & NPR3 (suggested, needs validation) & KCNA1 (suggested, needs validation) \\
\hline $\begin{array}{l}\text { Chromosomal } \\
\text { abnormalities }\end{array}$ & $\begin{array}{l}\text { Monosomy of } \\
\text { chromosome } 6 \text { (diploid } \\
\text { in older patients) }\end{array}$ & $\begin{array}{l}9 q \text { deletion, loss of } 10 q \text { and } 17 p \text {, gains of } 3 q \text { and } \\
9 p\end{array}$ & $\mathrm{i} 17 \mathrm{q}, 1 \mathrm{q}$ gain, loss of $5 q$ and $10 q$ & $\begin{array}{l}i 17 q, \text { loss of } 8,10,11 \text { and gain of } 4,7 \\
17, \text { and } 18\end{array}$ \\
\hline Genetics & $\begin{array}{l}\text { CTNNB1, DDX3X, } \\
\text { SMARCA4, TP53 and } \\
\text { KMT2D }\end{array}$ & $\begin{array}{l}\text { PTCH1, TP53 (high prognostic impact), KMT2D, } \\
D D X 3 X, M Y C N, B C O R, L D B 1, G L I\end{array}$ & $\begin{array}{l}\text { MYC, OTX2, SMARCA4, NOTCH, } \\
\text { TGF- } \beta\end{array}$ & $M Y C N, C D K N A$, SNCAIP duplications \\
\hline Epigenetics & $\begin{array}{l}\text { SRFP family (inhibitors } \\
\text { of WNT signaling } \\
\text { pathway) }\end{array}$ & $\begin{array}{l}\text { MLL2/KMT2D \& MLL3/KMT2C (promote } \\
\text { H3K4me2/3), SMARCA4 }\end{array}$ & H3K27, H3K4, BRD/BRD & H3K27, H3K4, KDM family \\
\hline Diagnosis & $\begin{array}{l}\text { Exon } 3 \text { sequencing for } \\
\text { CTNNB1 or both nuclear } \\
\beta \text {-catenin (IHC) and } \\
\text { monosomy } 6 \text { (FISH) }\end{array}$ & $\begin{array}{l}\text { Gene expression/methylation profiling and/or IHC } \\
\text { for filamin A and YAP1 +/- GAB1 }\end{array}$ & $\begin{array}{l}\text { Genome wide methylation, } \\
\text { expression array }\end{array}$ & $\begin{array}{l}\text { Genome wide methylation, } \\
\text { expression array }\end{array}$ \\
\hline $\begin{array}{l}\text { Prognosis (5-year } \\
\text { survival) }\end{array}$ & $97 \%(\alpha) \quad 100 \%(\beta)$ & $69.8 \%(\alpha) \quad 67.3 \%(\beta) \quad 88 \%(\gamma)$ & $66.2 \%(\alpha) \quad 55.8 \%(\beta) \quad 41.8 \%(\gamma)$ & $66.8 \%(\alpha) \quad 75.4 \%(\beta)$ \\
\hline $\begin{array}{l}\text { Possible targeted } \\
\text { therapies }\end{array}$ & $\begin{array}{l}\text { Trichostatin A (HDAC } \\
\text { inhibitor), other small } \\
\text { molecules to inhibit } \\
\text { WNT pathway in } \\
\text { preclinical studies }\end{array}$ & $\begin{array}{l}\text { Vismodegib, arsenic trioxide, bromdomain } \\
\text { inhibitors, aurora kinase inhibitors }\end{array}$ & $\begin{array}{l}\text { Bromdomain inhibitors, HDAC } \\
\text { inhibitors, PI3K inhibitors }\end{array}$ & None \\
\hline $\begin{array}{l}\text { Genetic } \\
\text { predisposition }\end{array}$ & $\begin{array}{l}\text { APC (germline), most } \\
\text { tumors lack CTNNB1 } \\
\text { mutation }\end{array}$ & SUFU, PTCH1, TP53, PALB2, and BRCA2 & $P A L B 2$ and $B R C A 2$ (rare) & PALB2 and $B R C A 2$ (rare) \\
\hline Other & Increased permeability of & BBB, excellent outcome even with residual tumors & & \\
\hline
\end{tabular}

and are not readily available in most of the centers. A reliable and more accessible way to diagnose this subgroup is via identification by two markers performed in accredited diagnostic laboratories: nuclear $\beta$-catenin accumulation, monosomy 6 (whole chromosome loss) by FISH [48,50,51]. Relying solely on the positivity of immunohistochemistry for beta-catenin may lead to an incorrect diagnosis of a WNT subgroup due to patchy nuclear accumulation in some WNT cases. This applies also to monosomy 6, which can be occasionally observed in other subgroups [48-50].

The WNT-MB was molecularly separated into two subgroups: WNT- $\alpha(70 \%)$ and WNT- $\beta(30 \%)$. The former subgroup occurs mostly in children with ubiquitous monosomy 6 , unlike the latter group in which most of the patients are adults and chromosome 6 is diploid [28]. 
Prognosis in patients with WNT MB is generally excellent even in the presence of somatic TP53 mutation, incomplete resection and/or metastatic disease at presentation [48,52-54]. There was no significant difference in survival between WNT- $\alpha$ and WNT- $\beta$ [28]. With current therapy, 5-year survival exceeds $90 \%$ in patients below 16 years of age, older patients have worse outcome and are not considered as a low risk group [28,48]. Increase in the permeability of the blood-brain barrier due to the secretion of WNT antagonists allows better penetrance of chemotherapy to the tumor site especially for medications that typically do not cross the blood-brain barrier [23,52,55], which might, in part, explain the outstanding outcome this subgroup.

Although MB patients are currently classified as either standard or high risk [3], nonmetastatic WNT MB has the most favorable outcome among MB subgroups and can be regarded as 'the low risk group'. Accordingly, studies are being designed to reduce the intensity of therapy in order to minimize long-term toxicities, especially effects on intelligence quotient and cognitive functioning. The ongoing SIOP-PNET5 (NCT02066220) and SJMB12 (NCT01878617) studies are exploring the possibility of reducing craniospinal radiation therapy to 18 and 15 Gray respectively. Surgery is major part of MB treatment and is usually performed upfront prior to radiation therapy. The current practice favors complete tumor resection to attain a better outcome. This is sometimes achieved at a high cost due to the damage caused by the surgery, especially when the tumor is in critical sites like the brainstem and/or infiltrating through foramen of Lushcka. As shown by Thompson et al., WNT MB has an excellent outcome, even when $<90 \%$ resection is achieved [56]. This permits less aggressive approach, protecting the patient functionality without compromising the outcome.

\section{Targeted therapy for WNT-MB}

Small molecules that can inhibit the WNT pathway and are ideally less toxic and more effective for specific patient subgroups seem to be an ideal and promising target to pursue. Direct targeting of WNT signaling has been difficult, largely owing to the lack of pathway-specific targets and the potential redundancy of many pathway components. Nonetheless, with the help of high-throughput screening of WNT-activated luciferase reporter assays, multiple molecules that can inhibit the WNT pathway have been discovered. These molecules are undergoing preclinical testing [46]. The use of histone deacetylases (HDAC) inhibitors, which disrupts chromatin remodeling, has been suggested in WNT MB patients as it can disrupt chromatin remodeling [36].

\section{SHH-subgroup}

The SHH-MB constitutes $28-30 \%$ of all MBs and has a bimodal age distribution (infants $<4$ years and adults $>16$ years old). Metastatic disease at diagnosis occurs in $15-25 \%$ of cases. Histologically, ND variant is the most common $(\sim 30 \%)$ and is almost exclusive for SHH-MB, followed by classic and large cell anaplastic subtypes) $[26,34,41,42]$. Immunoreactivity for filamin A and YAP1 in SHH tumors are usually strong and widespread. Cytoplasmic GAB1 immunoreactivity ranges from weak to absent [40]. Hence, immunostaining is a good option to identify SHH subgroup if gene expression and methylation profiling are not available or unaffordable.

This MB subgroup was named after the SHH signaling pathway, which is one of the major signaling networks that regulates the key events during developmental processes [21,57]. Deregulations in the SHH signaling pathway may lead to birth defects, tissue regeneration, stem cell renewal and cancer growth [57]. In the brain, granule cell (GC) progenitors migrate from the upper rhombic lip to the external granule layer, where they initiate proliferation in the outer external granule layer. The $\mathrm{SHH}$, secreted by Purkinje neurons starting from embryonal week 18, is a critical mitogen regulating GC progenitor proliferation. Hence, SHH tumors mainly arise in the hemispheric areas, although cases are reported in the midline [58,59]. Germline mutations in SHH receptor PTCH was first linked with $\mathrm{MB}$ as a result of the finding that patients with Gorlin syndrome were found to have a strong predisposition to the disease [60]. This opened the door to the discovery of multiple somatic mutations and driver genes in SHH-MB through transcriptional profiling. The most prominent driver genes are PTCH1 (28\%); TP53 (13.6-21\%, almost exclusively in patients 5-18 years old); KMT2D (12.9\%); DDX3X (11.7\%); MYCN amplification (8.2\%); BCOR (8\%); LDB1 (6.9\%); TCF4 (5.5\%) and GLI2 amplification (5.2\%) [13,15,17,21,47,52,61,62]. The MYCN is almost exclusively associated with deletions of chromosome $9 \mathrm{q}$, which is also the location of the PTCH1 gene (9q22). Less frequently than chromosome 9 loss, SHH-MB might have $10 \mathrm{q}$ and $17 \mathrm{p}$ losses and gains of $3 q$ and $9 \mathrm{p}$ [13].

Since the discovery of the four core MB subtypes in 2010, molecular subgrouping has remarkably revolutionized, ending up with 12 molecular subtypes. Cavalli et al. analyzed 763 primary MB samples collected from the Medulloblastoma Advanced Genomics International Consortium and proved that SHH-MB can now be divided into four subgroups (SHH- $\alpha, \beta, \gamma$ and $\delta$ ) reflecting distinct somatic copy-number aberrations, activated pathways 
and clinical outcomes [28]. The SHH $\alpha$ affect children aged 3-18 years and have the worst prognosis in this subgroup. Histologically, it can be of LCA or ND subtypes. They are enriched for MYCN, GLI2, YAP1 amplifications, TP53 mutations and copy number alterations (9q, 10q, 17p losses). When compared with other SHH subgroups and WNT-MB, TP53 mutation was prognostically significant in $\mathrm{SHH} \alpha$ subgroup. Metastatic disease occurred in around $70 \%$ of cases at diagnosis.

Infant SHH-MBs (age $<3$ years) mainly belong to $\beta$ and $\gamma$ subgroups, although with dissimilar molecular profiles and outcomes. Total of $25 \%$ of SHH $\beta$ harbor focal PTEN deletions and have multiple focal amplifications. A third of $\mathrm{SHH} \beta$ are metastatic at diagnosis, which explains the dismal outcome (67\% 5-year survival) in this subgroup. Unlike SHH $\beta$, SHH $\gamma$ have a better outcome ( $88 \%$ 5-year survival), balanced genome and no recurrent amplifications. The ND and MBEN are the main subtypes (all MBEN MB are SHH $\gamma$ ). The SJYC07 (NCT00602667) Phase II study showed consistent results with the Cavalli group where infant SHH-MB (iSHH) was separated into two subgroups; iSHH-I and iSHH-II based on the methylation subgroup analysis in relation to the EFS. The iSHH-I was enriched for SUFU aberrations and chromosome 2 gain. The 5-year progression free survival (PFS) was 75 versus $28 \%$ in iSHH-II versus iSHH-I respectively. This is very important to note as iSHH-II group can be cured with less aggressive therapy (craniospinal radiotherapy, myeloablative chemotherapy or intraventricular therapy), while iSHH-I require aggressive treatment approaches [63].

Adults dominate the SHH- $\delta$ subgroup with favorable outcome (88.5\%, 5-year survival) and less than $10 \%$ cases with metastatic disease at presentation. This subgroup is enriched with ND subtype and TERT promotor mutations [64].

Schwalbe $e$ al. published the results of their study based on the analysis of 428 centrally reviewed MB cases from patients aged 0-16 years from multiple European centers. They categorized SHH patients into two groups (SHH infant and SHH child). Both groups were enriched in SUFU and PTCH1 mutations and had a 10-year overall survival (OS) of 58 and $48 \%$ respectively. The SHH child group showed mutations in TP53, TP53 GLI and TERT, in addition to SUFU and PTCH1 as well as $9 \mathrm{p}$ gain, $1 \mathrm{p}$ and $9 \mathrm{q}$ deletions [27].

\section{Targeted therapy for $S H H M B$}

The PBTC-025 trial showed promising responses with vismodegib (GDC-0449, Genetech Corporation, MA, USA), a smoothened protein inhibitor, in a subset of adult patients with recurrent SHH-MB. However, responses were shortlived as patients developed resistance to the drug after prolonged exposure. Additionally, patients with immature bony skeletons develop premature fusions of the epiphyses; hence, SMO inhibitors are only indicated with mature skeletons. Patients with downstream SUFU and GLI2 mutations will not benefit from such therapy [31]. The currently ongoing St Jude SJMB12 (NCT01878617) incorporates vismodegib in the treatment of SHH-MB patients. Pending the results of the SJMB12 study, subsets of SHH-MB with poor prognosis (TP53 [germline and somatic], SUFU, GLI2 mutations), pose a great challenge and mandate newer therapeutic options to improve their prognosis. Arsenic trioxide (GLI2 inhibitor) and bromdomain (BRD) inhibitors, targeting SHH transcriptional output, seem to have a promising potential $[65,66]$. Aurora-kinase inhibitors have been suggested as possible therapeutic option for tumors with $M Y C N$ mutation [67]. All these drugs are still in the preclinical testing phase and require validation in Phase I trials in patients with recurrent disease.

\section{G3-Subgroup}

The G3-MB accounts for 25-28\% of all MBs [34,42]. It occurs predominantly in males (2:1 male to female ratio). Only two histologic variants are encountered in G3-MB, classic (most common) and LCA. Metastatic disease is very common at diagnosis affecting up to $45 \%$ of cases $[17,21,42]$. Most of the patients recur with metastatic dissemination while tumor bed is free of disease [68]. The proposed cells of origin for this subgroup are cerebellar granule-neuron precursor cells of the external granule-cell layer and are usually located in the midline filling the fourth ventricle $[42,43,69]$.

They display cytoplasmic immunoreactivity for $\beta$-catenin and do not stain for GAB1 and YAP1 [40]. Immunohistochemistry for natriuretic peptide receptor-3 has been proposed by several studies $[13,70]$.

Unlike previous data showing that less than a third of G3-MB had at least one probable driver gene [54,70,71], recent evidence based on a large dataset has confirmed that $76 \%$ of G3-MB can be related to a potential driver gene [30].

The most common recurrent somatic genetic mutations occurring in G3-MB are the mutually exclusive $M Y C$ and OTX2 (16-20 and 7.7\% respectively) [42,72]. The MYCN amplification is detected in 5\% of G3-MB [30,34]. 
Structural variants, including deletions, duplications and inversions, place the growth factor independent family of proto-oncogenes GFI1 and GFIIB next to active enhancer elements, resulting in their aberrant activation in $41 \%$ of the cases $[30,34,73]$. The most frequently mutated gene in G3-MB is SMARCA4 (11\%). Significant overrepresentation of genes involved in the notch and tumor growth factor- $\beta$ (TGF- $\beta$ ) signaling pathways are currently well documented in G3-MB [30,74,75].

G3-MB has a remarkably low number of recurring single nucleotide variants and is represented by a series of recurrent DNA copy number gains or losses of chromosomal arms or of whole chromosomes [36,48]. Isochromosome 17 (iq17) is detected in about $25 \%$ of the cases. Gain of chromosome 1q and/or loss of chromosome 5q and 10q is also associated with this subgroup [21,34,58,74].

Identification of this subgroup is best performed using either genome-wide methylation, expression array methods or limited panels (22 NanoString signature) [76].

Cavalli et al. recently subcategorized 144 G3-MB into G3- $\alpha$ (46.5\%), G3- $\beta$ (25.7\%) and G3- $\gamma$ (27.8\%) subgroups with distinct copy-number aberrations, activated pathways and clinical outcomes. The G3- $\alpha$ mostly affects infants and young children up to 10 years of age, with $60 \%$ of infants in G3-MB belonging to this subgroup. Metastatic disease is present in $43.4 \%$ of cases at diagnosis, and the 5 year OS is $66.2 \%$. Chromosomal abnormalities frequently encountered in this subgroup are chromosomes 8, 10 and 11 loss, chromosome 7 gain, and i17q. MYC amplification occurs in $7.1 \%$ of the cases. The G3- $\beta$ commonly occurs in older children and is less frequently metastatic at diagnosis (20\%). It is enriched with OTX1 gain and DDX31 loss on chromosome 9 and a high expression of GFI1/1B. A 5-year OS is $55.8 \%$ [28].

The G3- $\gamma$ has the worst outcome in this group with a 5 -year survival of $41.9 \%$, and almost $40 \%$ of patients are metastatic at diagnosis. This group has a similar age range as G3- $\alpha$. It is enriched for iq17 (25\%) and frequently harbor $M Y C$ amplification (20.8\%) [28]. Interestingly, G3- $\gamma$ has a poor prognosis regardless of the MYC status, amplifying the role of other factors related to bad prognosis and questioning the previous observations that $M Y C$ nonamplified G3 MB has a better prognosis [15]. Prognosis of G3 patients in the SJYC07 was poor in both intermediate and high risk groups (5-year PFS 15.4 vs $0.0 \%$ respectively, p-value $=0.22$ ) [63].

\section{Therapeutic options for G3-MB}

Finding new effective therapies for G3-MB is of high importance given the overall dismal prognosis. Highthroughput screening has identified pemetrexed and gemcitabine as possibly effective against G3-MB [32] and is currently being tested by the ongoing St Jude trial (SJMB12, NCT01878617). The BRD inhibitors, interfering with $M Y C$-associated transcriptional activity, seem to be a promising option [77]. Targeting the histone methylases EZH2 and KDM6A represents possible future targets [24]. The HDAC inhibitors potently inhibit MYC-driven $\mathrm{MB}$ cell growth in vitro and synergize with PI3K inhibitors to inhibit tumor growth in vivo [78,79].

\section{G4-subgroup}

The G4-MB is the most common subtype of MBs. It rarely arises in infants and occurs much more frequently in males than in females (3:1 ratio) across all age groups (median age $=9$ years). It accounts for $40-45 \%$ of childhood MBs [21,42,52,72]. Classic histology is the commonest finding in G4-MB with LCA less frequently encountered [72]. They are usually located in the midline filling the fourth ventricle [59]. The KCNA1 has been suggested as a possible immunohistochemical marker for the diagnosis of G4-MB; however, validation of this test is required [13,15]. Similar to G3, G4 has been recently subdivided into three subgroups, based on the analysis of 326 G4-MB, into G4- $\alpha$ (30\%), G4- $\beta$ (33.4\%) and G4- $\gamma(36.6 \%)$. The G4- $\alpha$ is enriched for MYCN (16.7\%) and CDKNA amplifications, as well as $8 \mathrm{p}$ loss and $7 \mathrm{q}$ gain. The $M Y C N$ amplification is not associated with an inferior outcome as in $\mathrm{SHH}$ group [34]. The G4- $\beta$ is strongly enriched for synuclein, $\alpha$ interacting protein (SNCAIP) duplications (15\%). Similar to G4- $\alpha$, G4- $\gamma$ is enriched for with $8 \mathrm{p}$ loss, $7 \mathrm{q}$ gain and CDKNA amplifications. The i17q is encountered in all G4 subgroups, with the biggest share for G4- $\beta$ ( 80 vs $41 \%$ in G4- $\alpha$ and $26 \%$ in G4- $\gamma$ ) [28]. Metastatic disease at diagnosis is almost identical in the three subgroups ( $40 \%)$. Analysis of outcome (5-year OS) did not show significant difference between the three groups $(68.8,75.4$ and $82.5 \%$ respectively). In general, G4-MB patients with metastatic disease at diagnosis have a higher risk of relapse, except in the presence of either whole chromosome 11 loss or chromosome 17 gain [34]. Infant G4 seems to experience poor survival as compared with children and adults [80]. In the SJYC07 trial, G4 group was divided into intermediate and high risk subgroups. The 5-year PFS was 16.7 and $0.0 \%$ respectively. When compared with the SHH group, the results were inferior $(13.3$ vs $51.1 \%$ respectively); however, not statistically significant $(\mathrm{p}$-value $=0.095)$ [63]. 


\section{Therapeutic options for $G 4-M B$}

Unfortunately, unlike with other MB subgroups, there are no currently active clinical or preclinical studies for G4-MB. The molecular overlap between G3 and G4-MB might be of use in extrapolating future therapies for this subgroup [34,42].

The good news about breaking down the currently overlapping G3 and G4 MB into several subgroups with distinct molecular characteristics, clinical behavior and prognosis is that it opens the door to reclassifying them into more distinct subgroups. This should eventually reflect on improving our therapeutic strategies and survival rates.

\section{Surgical resection by molecular subgroup}

Analysis of degree of resection of $787 \mathrm{MB}$ patients by molecular subgroup showed a benefit of in progression free survival (PFS) of GTR over STR but no significant PFS or EFS benefit between GTR and NTR and no survival benefit to greater extent of resection for patients with WNT, SHH and G3 subgroups. Group 4 patients had increased risk of progression with STR as compared with GTR and NTR, especially in metastatic patients [56]. This study evaluated the largest cohort of patients for the role of extent of resection in the treatment and outcome of MB subgroups. Although it was limited by the absence of central radiology review, it highlights the importance of individualizing the surgical approach in MB patients to avoid surgical morbidity and maintain adequate cure rates.

\section{Epigenetics in MB}

Epigenetics involves changes in the gene function that are mitotically and/or meiotically heritable and that do not involve changes in the individual's DNA sequence. This is achieved through DNA methylation, histone modifications, chromatin remodeling, microRNAs and LncRNAs [33,81]. As mentioned in this paper, childhood MBs harbor multiple genetic mutations leading to tumorigenesis. However, there are subtypes (mainly G3 and G4) that have no reported mutations, suggesting the role of epigenetic dysregulation [33]. Hypermethylation of $5^{\prime}$-Cphosphate-G-3' (CpG) dense promotors (CDKN2A, H1C1 and RASSF1) leads to the silencing of tumor suppressor genes, including PTCH1, SFRP family and ZIC2 [33,37,82]. The DNA methylation inhibitor like 5-azacytidine has been suggested as a therapeutic option $[33,83]$. Somatic mutations and copy number aberrations in HDACs, demethylases, histone lysine methyltransferases, as well as members of the polycomb transcriptional repressor complex (PRC2 and PRC1) have been identified across all four MB subgroups. Aberrant histone methylation at $\mathrm{H} 3 \mathrm{~K} 27$ and H3K4 are found in G3 and G4 MB. Mutations in KDM family members are among the most common recurrent events in G4 MB. The BRD and extraterminal (BET)-containing proteins control MYC levels, a common driver in G3 MB. Small molecule BET inhibitors are currently being studied in preclinical settings [33,77,84,85]. The HDAC inhibitors such as valproic acid and trichostatin might have a role in MB treatment [33]. miRNAs and long noncoding RNAs (LncRNAs) are reported to have a role in MB. miRNAs can either be suppressed (miR-124) or overexpressed (miR-17 192). The LncRNAs regulate the nuclear structure, gene expression and post-transcriptional processing $[33,86]$.

\section{Genetic predisposition in MB}

Even prior to the discovery of the four $\mathrm{MB}$ subgroups, $\mathrm{MB}$ has been observed in conjunction with several rare disorders, including Gorlin syndrome (associated with mutations in SUFU and PTCH1), Li-Fraumeni syndrome (TP53), Fanconi anemia (BRCA2) and $A P C$-associated polyposis conditions [14,87-89]. In a very recent study, Waszak et al. analyzed blood samples for germline mutations from 1022 patients with MB (673 from previous retrospective cohorts and 349 from prospective studies) and matched them with 800 available tumor samples for 110 cancer predisposition genes. Damaging germline mutations were identified in $11 \%$ of the retrospective cohort and results were replicated in the prospective study. The most relevant genes identified were APC, BRCA2, PALB2, PTCH1, SUFU and TP5 [25]. The prevalence of the above six genes was $6 \%$ overall and as high as $20 \%$ in SHH-MB. Three important findings were documented in this study: G3 and G4 subgroups were rarely associated with germline mutations and the absence of biallelic germline mutations in mismatch repair genes (only one patient harbored heterozygous germline mutation in $M S H 6$ gene) [25]. The authors conclude that patients with WNT and SHH MBs are most commonly associated with a genetic predisposition, and half of the identified patients lacked relevant family history. This mandates genetic counseling for those patients for earlier detection and management of associated malignancies. 


\section{Molecular genetics at recurrence}

Despite the advances in MB therapy [3,5,90], a third of patients suffer from relapse. Outcome in relapsed MB (rMB) is dismal with less than $10 \%$ survival rates at best in patients who have received prior irradiation. Therapy in $\mathrm{rMB}$ is nonuniform, ranging from palliative therapy in most of the cases, to high dose chemotherapy [81,91,92]. The results of the COG trial for rMB ACNS0821 (NCT01217437) were disappointing with a median event-free survival (EFS) and OS of 6 and 19 months respectively [93]. Ramaswamy et al. showed in a group of 34 matched samples of newly diagnosed and $\mathrm{rMB}$ patients that $\mathrm{MB}$ does not change subgroup at recurrence. Subgroup analysis in this study demonstrated that SHH-MB mostly recurs locally in the tumor bed, while G3 and G4-MB mostly recur at distant sites with devoid tumor bed [68]. Similarly, Hill et al., in a cohort of 29 recurrent tumors with matched samples at diagnosis, demonstrated that MB does not change subgroup at relapse. However, they showed that relapsed tumors commonly acquire combined MYC family amplifications and P53 pathway defects (32\%). This combination was encountered across all subgroups, although most commonly seen in SHH (60\%) and least encountered in G4 (11\%) subgroups [67]. These findings are of great essence to the future trials which can be directed based on tumor biology and patterns of recurrence.

\section{Conclusion}

The MB is not a single disease anymore, it is a group of four diseases characterized by genomic, biologic and clinical diversity even within the same group. As discussed earlier, group or subgroup targeted therapies are yet to be investigated; nonetheless, treatment decisions based on specific subgroup characteristics can be made and can readily affect patient survival and long-term side effects.

\section{Future perspective}

It is amazing how much MB diagnosis and treatment have advanced since the early years of the 20th century. The discovery of the four robust molecular subgroups, and the recent refinements has transformed our understanding of this disease. Unfortunately, treatment-related morbidity and mortality are still a significant burden. Additionally, outcomes are still suboptimal when it comes to high risk, metastatic and relapsed patients. The immense amount of genetic and epigenetic findings and their correlation with multiple factors including age, anatomic location, risk stratification, surgical approach and patterns of relapse and possible therapeutic targets should be the foundation of future trials that will hopefully deliver a perfectly crafted individualized therapy, providing best outcomes and minimizing treatment side effects. In clinical practice, subgrouping should soon become a standard of care and should be strictly performed in certified laboratories following international guidelines to avoid inaccurate grouping leading to catastrophic consequences. Validating immunohistochemical tests to replace the more advanced expensive genomic testing will ensure accurate subgrouping in centers with high load and low budgets (low and middle income countries).

\section{Acknowledgments}

We would like to thank Mrs Fatimah Shamdeen for the editorial review. Special thanks to Mr Maarouf Chebbo for his assistance with the graphics in Table 1 and Figure 1.

\section{Financial \& competing interest disclosure}

The authors have no relevant affiliations or financial involvement with any organization or entity with a financial interest in or financial conflict with the subject matter or materials discussed in the manuscript. This includes employment, consultancies, honoraria, stock ownership or options, expert testimony, grants or patents received or pending, or royalties.

No writing assistance was utilized in the production of this manuscript.

\section{Open access}

This work is licensed under the Creative Commons Attribution-NonCommercial 3.0 Unported License. To view a copy of this license, visit http://creativecommons.org/licenses/by-nc-nd/3.0/ 
- Until recently, medulloblastoma (MB) was classified according to histologic characteristics.

- Current standard of care for MB incorporates maximum safe surgical resection, irradiation and chemotherapy.

- In 2010, four molecular subgroups (Wingless [WNT], Sonic Hedgehog [SHH], group 3 and group 4) were identified and validated by all major research groups.

- WHO CNS classification included the four groups for the first time in 2016.

- Groups were further refined into multiple subgroups reflecting genomic, biologic and clinical heterogeneity.

- Epigenetic dysregulation plays a role in MB.

- Recurrent tumors retain their original molecular characteristics.

- Genetic counseling is important especially for WNT and SHH-MB.

WNT subgroup

- A total of $10-15 \%$ of $\mathrm{MB}$, peak age 10-12 years, classic histology most common, lower rhombic lip progenitor cell of the dorsal midbrain are the cells of origin, usually located centrally and infiltrating foramen of Lushcka.

- Presence of nuclear $\beta$-catenin and monosomy of chromosome 6 is diagnostic.

- Less aggressive surgery and lower doses of radiation are required.

- Excellent prognosis even with metastatic disease and somatic TP53 mutation.

SHH subgroup

- Total of $28-30 \%$ of MB, bimodal age distribution, nodular desmoplastic histology most common. Cerebellar GCP of the EGC are the cells of origin. Usually located in the cerebellar hemispheres.

- Immunohistochemistry for filamin A and YAP 1 are diagnostic.

- PTCH1 and TP53 mutations have high prognostic impact.

- Prognosis is variable by subgroup and dependent on multiple factors (intermediate to very poor).

- Infant SHH-MB may have good or poor outcome based on methylation profiling.

- Targeted therapies are under investigation.

Group 3

- A total $25 \%$ of MB, mostly affects infants and young children. Classic histology is most common.

- Usually located in the midline filling the fourth ventricle.

- High rate of metastatic disease at diagnosis.

- High rate of $i 17 q$ and $M Y C$ amplification.

- Diagnosis made by genome wide methylation and expression array.

- Prognosis generally poor.

- Suggested targeted therapies, no current clinical trials.

Group 4

- The most common group (40-45\%), can occur at any age. Classic histology is most common. Cell of origin is unknown.

- Usually located in the midline filling the fourth ventricle.

- High rate of metastatic disease at diagnosis.

- Intermediate to good prognosis depending on molecular subgroup.

- No possible targeted therapies identified.

\section{References}

Papers of special note have been highlighted as: $\bullet$ of interest; $\bullet \bullet$ of considerable interest

1. Ostrom QT, Gittleman H, Xu J et al. CBTRUS statistical report: primary brain and other central nervous system tumors diagnosed in the United States in 2009-2013. Neuro. Oncol. 18, v1-v75 (2016).

2. Smoll NR, Drummond KJ. The incidence of medulloblastomas and primitive neurectodermal tumours in adults and children. J. Clin. Neurosci. 19(11), 1541-4 (2012).

3. Gajjar A, Chintagumpala M, Ashley D et al. Risk-adapted craniospinal radiotherapy followed by high-dose chemotherapy and stem-cell rescue in children with newly diagnosed medulloblastoma (St Jude Medulloblastoma-96): long-term results from a prospective, multicentre trial. Lancet Oncol. 7(10), 813-820 (2006).

4. Hughes EN, Shillito J, Sallan SE, Loeffler JS, Cassady JR, Tarbell NJ. Medulloblastoma at the joint center for radiation therapy between 1968 and 1984. The influence of radiation dose on the patterns of failure and survival. Cancer 61(10), 1992-1998 (1988).

5. Packer RJ, Gajjar A, Vezina G et al. Phase III study of craniospinal radiation therapy followed by adjuvant chemotherapy for newly diagnosed average-risk medulloblastoma. J. Clin. Oncol. 24(25), 4202-4208 (2006).

- Landmark paper showing that decreasing the craniospinal radiation dose in average risk patients does not compromise the outcome and reduced the toxicity.

6. Rutka JT, Hoffman HJ. Medulloblastoma: a historical perspective and overview. J. Neurooncol. 29(1), 1-7 (1996). 
7. Mulhern RK, Horowitz ME, Kovnar EH, Langston J, Sanford RA, Kun LE. Neurodevelopmental status of infants and young children treated for brain tumors with preirradiation chemotherapy. J. Clin. Oncol. 7(11), 1660-1666 (1989).

8. Jenkin D, Danjoux C, Greenberg M. Subsequent quality of life for children irradiated for a brain tumor before age four years. Med. Pediatr. Oncol. 31(6), 506-511 (1998).

9. Slampa P, Pavelka Z, Dusek L et al. Longterm treatment results of childhood medulloblastoma by craniospinal irradiation in supine position. Neoplasma 54(1), 62-67 (2007).

10. Palmer SL, Reddick WE, Gajjar A. Understanding the cognitive impact on children who are treated for medulloblastoma. J. Pediatr. Psychol. 32(9), 1040-1049 (2007).

11. Ris MD, Packer R, Goldwein J, Jones-Wallace D, Boyett JM. Intellectual outcome after reduced-dose radiation therapy plus adjuvant chemotherapy for medulloblastoma: a Children's Cancer Group study. J. Clin. Oncol. 19(15), 3470-3476 (2001).

12. Palmer SL, Goloubeva O, Reddick WE et al. Patterns of intellectual development among survivors of pediatric medulloblastoma: a longitudinal analysis. J. Clin. Oncol. 19(8), 2302-2308 (2001).

13. Northcott PA, Korshunov A, Witt $\mathrm{H}$ et al. Medulloblastoma comprises four distinct molecular variants. J. Clin. Oncol. 29(11), 1408-1414 (2011).

14. Taylor MD, Liu L, Raffel C et al. Mutations in SUFU predispose to medulloblastoma. Nat. Genet. 31(3), 306-310 (2002).

15. Cho YJ, Tsherniak A, Tamayo P et al. Integrative genomic analysis of medulloblastoma identifies a molecular subgroup that drives poor clinical outcome. J. Clin. Oncol. 29(11), 1424-1430 (2011).

16. Northcott PA, Rutka JT, Taylor MD. Genomics of medulloblastoma: from Giemsa-banding to next-generation sequencing in 20 years. Neurosurg. Focus 28(1), E6 (2010).

17. Kool M, Koster J, Bunt J et al. Integrated genomics identifies five medulloblastoma subtypes with distinct genetic profiles, pathway signatures and clinicopathological features. PLoS ONE 3(8) (2008).

18. Reardon DA, Michalkiewicz E, Boyett JM et al. Extensive genomic abnormalities in childhood medulloblastoma by comparative genomic hybridization. Cancer Res. 57(18), 4042-4047 (1997).

19. Mertens F, Johansson B, Mitelman F. Isochromosomes in neoplasia. Genes Chromosomes Cancer 10(4), 221-230 (1994).

20. Ellison D. Classifying the medulloblastoma: insights from morphology and molecular genetics. Neuropathol. Appl. Neurobiol. 28(4), 257-282 (2002).

21. Taylor MD, Northcott PA, Korshunov A et al. Molecular subgroups of medulloblastoma: the current consensus. Acta Neuropathol. 123(4), 465-472 (2012).

-• Following this paper, molecular subgrouping of medulloblastoma became widely accepted.

22. Patay XZ, Desain LA, Hwang SN, Coan A, Li Y, Ellison DW. MR imaging characteristics of wingless-type - subgroup. AJNR. Am. J. Neuroradiol. 36(12), 2386-93 (2015).

23. Phoenix TN, Patmore DM, Boop S et al. Medulloblastoma genotype dictates blood-brain barrier phenotype. Cancer Cell 29(4), 508-522 (2016).

24. Huang GH, Xu QF, Cui YH, Li N, Bian XW, Lv SQ. Medulloblastoma stem cells: promising targets in medulloblastoma therapy. Cancer Sci. 107(5), 583-589 (2016).

25. Waszak SM, Northcott PA, Buchhalter I et al. Spectrum and prevalence of genetic predisposition in medulloblastoma: a retrospective genetic study and prospective validation in a clinical trial cohort. Lancet Oncol. 19(6), 785-798 (2018).

26. Kool M, Jones DTW, Jäger N et al. Genome sequencing of SHH medulloblastoma predicts genotype-related response to smoothened inhibition. Cancer Cell 25(3), 393-405 (2014).

27. Schwalbe EC, Lindsey JC, Nakjang $S$ et al. Novel molecular subgroups for clinical classification and outcome prediction in childhood medulloblastoma: a cohort study. Lancet Oncol. 18(7), 958-971 (2017).

- Reclassification of medulloblastoma into seven clinically significant subgroups.

28. Cavalli FMG, Remke M, Rampasek L et al. Intertumoral heterogeneity within medulloblastoma subgroups. Cancer Cell 31(6), 737-754.e6 (2017).

29. Bavle A, Parsons DW. From one to many: further refinement of medulloblastoma subtypes offers promise for personalized therapy. Cancer Cell 31(6), 727-729 (2017).

30. Northcott PA, Buchhalter I, Morrissy AS et al. The whole-genome landscape of medulloblastoma subtypes. Nature 547(7663), 311-317 (2017).

31. Robinson GW, Orr BA, Wu G et al. Vismodegib exerts targeted efficacy against recurrent Sonic Hedgehog - subgroup medulloblastoma: results from Phase II Pediatric Brain Tumor Consortium studies PBTC-025B and PBTC-032. J. Clin. Oncol. 33(24), 2646-2654 (2015).

32. Morfouace $\mathrm{M}$, Shelat $\mathrm{A}$, Jacus $\mathrm{M}$ et al. Pemetrexed and gemcitabine as combination therapy for the treatment of group3 medulloblastoma. Cancer Cell 25(4), 516-529 (2014).

33. Roussel MF, Stripay JL. Epigenetic drivers in pediatric medulloblastoma. Cerebellum 17(1), 28-36 (2018). 
34. Gajjar A, Bowers DC, Karajannis MA, Leary S, Witt H, Gottardo NG. Pediatric brain tumors: Innovative genomic information is transforming the diagnostic and clinical landscape. J. Clin. Oncol. 33(27), 2986-2998 (2015).

35. Louis DN, Perry A, Reifenberger G et al. The 2016 World Health Organization classification of tumors of the central nervous system: a summary. Acta Neuropathol. 131(6), 803-820 (2016).

-• WHO classification incorporates molecular sugrouping of medulloblastoma.

36. Northcott PA, Jones DTW, Kool M et al. Medulloblastomics: the end of the beginning. Nat. Rev. Cancer 12(12), 818-834 (2012).

37. Shahi MH, Afzal M, Sinha S et al. Regulation of Sonic Hedgehog-GLI1 downstream target genes PTCH1, cyclin D2, plakoglobin, PAX6 and NKX2.2 and their epigenetic status in medulloblastoma and astrocytoma. BMC Cancer 10, 1-20 (2010).

38. Pai SG, Carneiro BA, Mota JM et al. Wnt/beta-catenin pathway: modulating anticancer immune response. J. Hematol. Oncol. 10(1), $1-12(2017)$.

39. Sengupta S, Pomeranz Krummel D, Pomeroy S. The evolution of medulloblastoma therapy to personalized medicine. F1000Research 6(0), 490 (2017).

40. Ellison DW, Dalton J, Kocak M et al. Medulloblastoma: clinicopathological correlates of SHH, WNT, and non-SHH/WNT molecular subgroups. Acta Neuropathol. 121(3), 381-396 (2011).

41. DeSouza R-M, Jones BRT, Lowis SP, Kurian KM. Pediatric medulloblastoma - update on molecular classification driving targeted therapies. Front. Oncol. 4(July), 1-8 (2014).

42. Gajjar AJ, Robinson GW. Medulloblastoma: translating discoveries from the bench to the bedside. Nat. Rev. Clin. Oncol. 11(12), 714-722 (2014).

43. Gibson P, Tong Y, Robinson G et al. Origins. Nature 468(7327), 1095-1099 (2011).

44. Juhnke BO, Mynarek M, Rutkowski S. Refining medulloblastoma subgroups. Lancet Oncol. 18(7), 847-848 (2017).

45. Clifford SC, Lusher ME, Lindsey JC et al. Wnt/Wingless pathway activation and chromosome 6 loss characterize a distinct molecular sub-group of medulloblastomas associated with a favorable prognosis. Cell Cycle 5(22), 2666-2670 (2006).

46. Ellison DW, Kocak M, Dalton J et al. Definition of disease-risk stratification groups in childhood medulloblastoma using combined clinical, pathologic, and molecular variables. J. Clin. Oncol. 29(11), 1400-1407 (2011).

47. Thompson MC, Fuller C, Hogg TL et al. Genomics identifies medulloblastoma subgroups that are enriched for specific genetic alterations. J. Clin. Oncol. 24(12), 1924-1931 (2006).

48. Ramaswamy V, Remke M, Bouffet E et al. HHS Public Access. 131(6), 821-831 (2017).

49. Pietsch T, Schmidt R, Remke M et al. Prognostic significance of clinical, histopathological, and molecular characteristics of medulloblastomas in the prospective HIT2000 multicenter clinical trial cohort. Acta Neuropathol. 128(1), 137-149 (2014).

50. Goschzik T, Zur Muhlen A, Kristiansen G et al. Molecular stratification of medulloblastoma: comparison of histological and genetic methods to detect Wnt activated tumours. Neuropathol. Appl. Neurobiol. 41(2), 135-144 (2015).

51. Gottardo NG, Hansford JR, McGlade JP et al. Medulloblastoma Down Under 2013: a report from the third annual meeting of the International Medulloblastoma Working Group. Acta Neuropathol. 127(2), 189-201 (2014).

52. Zhukova N, Ramaswamy V, Remke M et al. Subgroup-specific prognostic implications of TP53 mutation in medulloblastoma. J. Clin. Oncol. 31(23), 2927-2935 (2013).

53. Ramaswamy V, Nör C, Taylor MD. p53 and Medulloblastoma. Cold Spring Harb. Perspect. Med. 6(2), a026278 (2016).

54. Von Bueren AO, Kortmann RD, Von Hoff K et al. Treatment of children and adolescents with metastatic medulloblastoma and prognostic relevance of clinical and biologic parameters. J. Clin. Oncol. 34(34), 4151-4160 (2016).

55. Sidaway P. CNS cancer: Wnt affects blood-brain barrier permeabilty. Nat. Rev. Clin. Oncol. 13(6), 330 (2016).

56. Thompson EM, Hielscher T, Bouffet E et al. Prognostic value of medulloblastoma extent of resection after accounting for molecular subgroup: a retrospective integrated clinical and molecular analysis. Lancet Oncol. 17(4), 484-495 (2016).

- First study to assess extent of resection in relation to molecular subgroup.

57. Choudhry Z, Rikani AA, Choudhry AM et al. Sonic hedgehog signalling pathway: a complex network. Ann. Neurosci. 21(1), 28-31 (2014).

58. Liu C, Zong H. Developmental origins of brain tumors. Curr. Opin. Neurobiol. 22(5), 844-849 (2012).

59. Gibson P, Tong Y, Robinson G et al. Subtypes of medulloblastoma have distinct developmental origins. Nature 468(7327), 1095-1099 (2010).

60. Fujii K, Miyashita T. Gorlin syndrome (nevoid basal cell carcinoma syndrome): update and literature review. Pediatr. Int. 56(5), 667-674 (2014).

61. Schwalbe EC, Lindsey JC, Straughton D et al. Rapid diagnosis of medulloblastoma molecular subgroups. Clin. Cancer Res. 17(7), 1883-1894 (2011).

62. Northcott PA, Fernandez-L A, Hagan JP et al. The miR-17/92 polycistron is up-regulated in Sonic Hedgehog-driven medulloblastomas and induced by N-myc in Sonic Hedgehog-treated cerebellar neural precursors. Cancer Res. 69(8), 3249-3255 (2009). 
63. Robinson GW, Rudneva VA, Buchhalter I et al. Risk-adapted therapy for young children with medulloblastoma (SJYC07): therapeutic and molecular outcomes from a multicentre, Phase II trial. Lancet Oncol. 19(6), 768-784 (2018).

64. Remke M, Ramaswamy V, Peacock J et al. TERT promoter mutations are highly recurrent in SHH subgroup medulloblastoma. Acta Neuropathol. 126(6), 917-929 (2013).

65. Kim J, Aftab BT, Tang JY et al. Itraconazole and arsenic trioxide inhibit Hedgehog pathway activation and tumor growth associated with acquired resistance to smoothened antagonists. Cancer Cell 23(1), 23-34 (2013).

66. Tang Y, Gholamin S, Schubert $S$ et al. Epigenetic targeting of Hedgehog pathway transcriptional output through BET bromodomain inhibition. Nat. Med. 20(7), 732-740 (2014).

67. Hill RM, Kuijper S, Lindsey JC et al. Combined MYC and P53 defects emerge at medulloblastoma relapse and define rapidly progressive, therapeutically targetable disease. Cancer Cell 27(1), 72-84 (2015).

68. Ramaswamy V, Remke M, Bouffet $\mathrm{E}$ et al. Recurrence patterns across medulloblastoma subgroups: an integrated clinical and molecular analysis. Lancet Oncol. 14(12), 1200-1207 (2013).

69. Perreault S, Ramaswamy V, Achrol AS et al. MRI surrogates for molecular subgroups of medulloblastoma. Am. J. Neuroradiol. 35(7), 1263-1269 (2014).

70. Eberhart CG, Kratz J, Wang Y et al. Histopathological and molecular prognostic markers in medulloblastoma: c-myc, N-myc, TrkC, and anaplasia. J. Neuropathol. Exp. Neurol. 63(5), 441-449 (2004).

71. Pugh TJ, Weeraratne SD, Archer TC et al. Medulloblastoma exome sequencing uncovers subtype-specific somatic mutations. Nature 488(7409), 106-110 (2012).

72. Kool M, Korshunov A, Remke M et al. Molecular subgroups of medulloblastoma: an international meta-analysis of transcriptome, genetic aberrations, and clinical data of WNT, SHH, group 3, and group 4 medulloblastomas. Acta Neuropathol. 123(4), 473-484 (2012).

73. Northcott PA, Lee C, Zichner T et al. Enhancer hijacking activates GFI1 family oncogenes in medulloblastoma. Nature 511(7510), 428-434 (2014).

74. Northcott PA, Shih DJH, Peacock J et al. Subgroup-specific structural variation across 1000 medulloblastoma genomes. Nature 488(7409), 49-56 (2012).

75. Lin CY, Erkek S, Tong Y et al. Active medulloblastoma enhancers reveal subgroup-specific cellular origins. Nature 530,57 (2016).

76. Northcott PA, Shih DJH, Remke M et al. Rapid, reliable, and reproducible molecular sub-grouping of clinical medulloblastoma samples. Acta Neuropathol. 123(4), 615-626 (2012).

77. Bandopadhayay P, Bergthold G, Nguyen B et al. BET bromodomain inhibition of MYC-amplified medulloblastoma. Clin. Cancer Res. 20(4), 912-925 (2014).

78. Pei Y, Liu KW, Wang J et al. HDAC and PI3K antagonists cooperate to inhibit growth of MYC-driven medulloblastoma. Cancer Cell 29(3), 311-323 (2016).

79. Furchert SE, Lanvers-Kaminsky C, Jürgens H, Jung M, Loidl A, Frühwald MC. Inhibitors of histone deacetylases as potential therapeutic tools for high-risk embryonal tumors of the nervous system of childhood. Int. J. Cancer. 120(8), 1787-1794 (2007).

80. Shih DJH, Northcott PA, Remke M et al. Cytogenetic prognostication within medulloblastoma subgroups. J. Clin. Oncol. 32(9), 886-896 (2014).

81. Koschmann C, Bloom K, Upadhyaya S, Geyer JR, Leary SES. Survival after relapse of medulloblastoma. J. Pediatr. Hematol. Oncol. 38(4), 269-273 (2016).

82. Shahi MH, Afzal M, Sinha $S$ et al. Human hedgehog interacting protein expression and promoter methylation in medulloblastoma cell lines and primary tumor samples. J. Neurooncol. 103(2), 287-296 (2011).

83. Sharma S, Kelly TK, Jones PA. Epigenetics in cancer. Carcinogenesis 31(1), 27-36 (2009).

84. Delmore JE, Issa GC, Lemieux ME et al. BET bromodomain inhibition as a therapeutic strategy to target c-Myc. Cell 146(6), 904-917 (2011).

85. Henssen A, Thor T, Odersky A et al. BET bromodomain protein inhibition is a therapeutic option for medulloblastoma. Oncotarget4(11), 2080-2095 (2013).

86. Huarte M. The emerging role of IncRNAs in cancer. Nat. Med. 21, 1253 (2015).

87. Wolter M, Reifenberger J, Sommer C, Ruzicka T, Reifenberger G. Mutations in the human homologue of the Drosophila segment polarity gene patched $(\mathrm{PTCH})$ in sporadic basal cell carcinomas of the skin and primitive neuroectodermal tumors of the central nervous system. Cancer Res. 57(13), 2581-2585 (1997).

88. Li FP, Fraumeni JFJ. Rhabdomyosarcoma in children: epidemiologic study and identification of a familial cancer syndrome. J. Natl. Cancer Inst. 43(6), 1365-1373 (1969).

89. Basis M, Turcot OF. The molecular basis of turcot's syndromes. 332(13), 839-847 (2014). 
90. Rutkowski S, Von Hoff K, Emser A et al. Survival and prognostic factors of early childhood medulloblastoma: an international meta-analysis. J. Clin. Oncol. 28(33), 4961-4968 (2010).

91. Sabel M, Fleischhack G, Tippelt $S$ et al. Relapse patterns and outcome after relapse in standard risk medulloblastoma: a report from the HIT-SIOP-PNET4 study. J. Neurooncol. 129(3), 515-524 (2016).

92. Wetmore C, Herington D, Lin T, Onar-Thomas A, Gajjar A, Merchant TE. Reirradiation of recurrent medulloblastoma: does clinical benefit outweigh risk for toxicity? Cancer 120(23), 3731-3737 (2014).

93. Levy A, Krailo M, Chi S, Williams-Hughes C, Villaluna MB, Fouladi MGA. Temozolomide with irinotecan versus temozolomide, irinotecan plus bevacizumab for recurrrent/refractory medulloblastoma/CNS PNET of childhood, a COG randomized Phase II Screening Trial. Neuro Oncol. 19(Suppl. 6), 186 (2017). 
